\title{
Production and Quality Control of MWPC for the LHCb Muon System at CERN
}

\author{
A. F. Barbosa, R. Dumps, J. S. Graulich, A. Kachtchouk, L. Manhães de Andrade Filho, E. Polycarpo, W. Riegler, \\ B. Schmidt, T. Schneider, and V. Souvorov
}

\begin{abstract}
This work describes the production and quality control of the multi-wire proportional chamber for the LHCb muon detection system. The European Laboratory of Particle Physics (CERN) is in charge of the production of 134 chambers for the inner regions of the five Muon Stations. These chambers have demanding requirements on rate capability $\left(>100 \mathrm{kHz} / \mathrm{cm}^{2}\right)$ and efficiency $(>99 \%)$ in a short time window $(20 \mathrm{~ns})$, on overall response uniformity which has to be better than $30 \%$, and on the aging properties. The technical design of the chambers is briefly presented, and the production line is described together with the quality assurance and quality control programs. This includes fabrication of the panels; wire winding, gluing, and soldering; gap uniformity control; pitch and wire tension measurement; gas leak tests; high-voltage training; and tests under intense radiation. An original method developed for gas gain uniformity measurements is described in detail, and the obtained results are shown.
\end{abstract}

Index Terms-Gain measurement, production and quality control, proportional counters.

\section{INTRODUCTION}

$\mathbf{T}$ HE muon system of the Large Hadron Collider beauty experiment (LHCb) [1] at the European Laboratory of Particle Physics (CERN) is composed of five stations, named M1 to M5, interleaved with thick iron walls. Each station is divided in four regions, R1 to R4, defining increasing angles from the interaction point. All the systems but the first station will be equipped with four-gap multi-wire proportional chambers (MWPC). The first station is placed before the calorimeter. It will be equipped with gas electron multiplier (GEM) chambers [2] in R1 and with two-gap MWPCs in the other regions.

The LHCb muon trigger requires a coincidence of the five stations in a time window given by the $\mathrm{LHC}$ bunch crossing rate ( 40 $\mathrm{MHz}$ ). In order to achieve a muon trigger efficiency of at least $95 \%$, the efficiency has to be higher than $99 \%$ over the entire surface of each station. On the other hand, the chambers have to

Manuscript received November 15, 2004; revised October 22, 2005. This work was partially supported by CNPq, Brazil.

R. Dumps, J. S. Graulich, A. Kachtchouk, W. Riegler, B. Schmidt, and T. Schneider are with the European Laboratory of Particle Physics (CERN), CH-1211, Geneva 23, Switzerland (e-mail: Jean-Sebastien.Graulich@ cern.ch). A. F. Barbosa and L. Manhães de Andrade Filho are with the Brazilian Center for Physics Research (CBPF), 22290-180 Rio de Janeiro, Brazil.

É. Polycarpo was with the Brazilian Center for Physics Research (CBPF), 22290-180 Rio de Janeiro, Brazil. She is now with the Physics Institute of the Federal University of Rio de Janeiro, 21941-972 Rio de Janeiro, Brazil (e-mail: poly@if.ufrj.br).

V. Souvorov was with the European Laboratory of Particle Physics (CERN), CH-1211, Geneva 23, Switzerland. He is now with NIKHEF, 1009 DB Amsterdam, The Netherlands.

Digital Object Identifier 10.1109/TNS.2006.869837

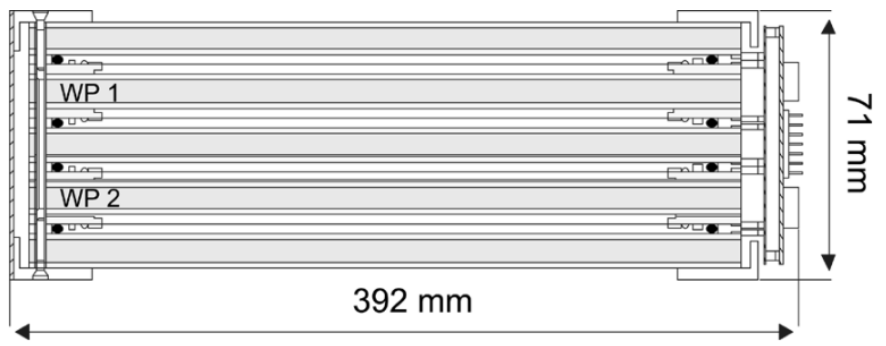

Fig. 1. Cross section of an MWPC for the LHCb Muon Station 3 (Region 1), equipped with the first stage of the FE board.

be operated at the lowest possible gas gain to reduce aging effects. In addition, the chambers will be exposed to a large radiation background, with rates ranging from a few hundred Hertz per square centimeter to more than $100 \mathrm{kHz} / \mathrm{cm}^{2}$ depending on the region. The signal will be read-out by the custom designed CERN and Rio Current Amplifier (CARIOCA) front-end chip [3].

The overall projective geometry of the detector has been designed to tag high $p_{T}$ muons at the first trigger level with a $p_{T}$ resolution of $20 \%$. Consequently, the chambers of each region have specific dimensions and segmentations that lead to significant differences in their design. The chambers produced at CERN will cover the inner regions M1-R2, M2-R1 and R2, M3-R1 and R2, M4-R1, and M5-R1, which add up to 134 chambers out of a total of 1440 . The nominal production rate is two chambers per week. In this paper, we focus on the M3 chambers for which the production is finished.

\section{Chamber Design And Production Procedures}

A cross section of the produced chambers is shown in Fig. 1. It is a stack of five panels, two of them equipped with $30 \mu \mathrm{m}$, goldcoated tungsten wires on both sides, with a pitch of $2 \mathrm{~mm}$. The gaps are hardwired together two by two before being connected to the front-end electronics (FEE) boards, defining two double gaps per chamber, which are eventually used in an OR after the FE chips. Table I summarizes the mechanical dimensions of the chambers produced at CERN.

The single gas gap thickness is $5 \mathrm{~mm}$. For the nominal gas mixture, $\mathrm{Ar} / \mathrm{CO}_{2} / \mathrm{CF}_{4}(40 / 55 / 5)$, the drift velocity is approximately $100 \mu \mathrm{m} / \mathrm{ns}$. The efficiency plateau starts at $2.55 \mathrm{kV}$ with an effective length of $250 \mathrm{~V}$. At $2.6 \mathrm{kV}$, the gain is about $2.510^{4}$. It doubles if the high voltage is increased by $110 \mathrm{~V}$. With a threshold of $8 \mathrm{fC}$, the time distribution RMS is $4 \mathrm{~ns}$ for the signal [4]. 
TABLE I

Mechanical Dimensions of THE MWPC PRoduced AT CERN FOR THE LHCb MUON SYSTEM

\begin{tabular}{|l|l|lll|}
\hline Ch. & $\begin{array}{l}\text { Panel Size } \\
\left(\mathrm{mm}^{2}\right)\end{array}$ & $\begin{array}{l}\text { Pad Area } \\
\left(\mathrm{mm}^{2}\right)\end{array}$ & $\begin{array}{l}\text { WireLength } \\
(\mathrm{mm})\end{array}$ & $\begin{array}{l}\text { Wire } \\
\text { Group }\end{array}$ \\
\hline M1-R2 & $566 \times 282$ & $20 \times 25$ & 200 & 10 \\
\hline M2-R1 & $390 \times 335$ & $38 \times 31.6$ & 252.8 & $3-4$ \\
\hline M2-R2 & $694 \times 335$ & $76 \times 31.6$ & 252.8 & $6-7$ \\
\hline M3-R1 & $414 \times 355$ & $41 \times 34.1$ & 272.8 & $3-4$ \\
\hline M3-R2 & $742 \times 355$ & $82 \times 34.1$ & 272.8 & $6-7$ \\
\hline M4-R1 & $438 \times 375$ & $29.3 \times 36.6$ & 292.8 & $14-15$ \\
\hline M5-R1 & $462 \times 395$ & $31.3 \times 39.1$ & 312.8 & $15-16$ \\
\hline
\end{tabular}

414

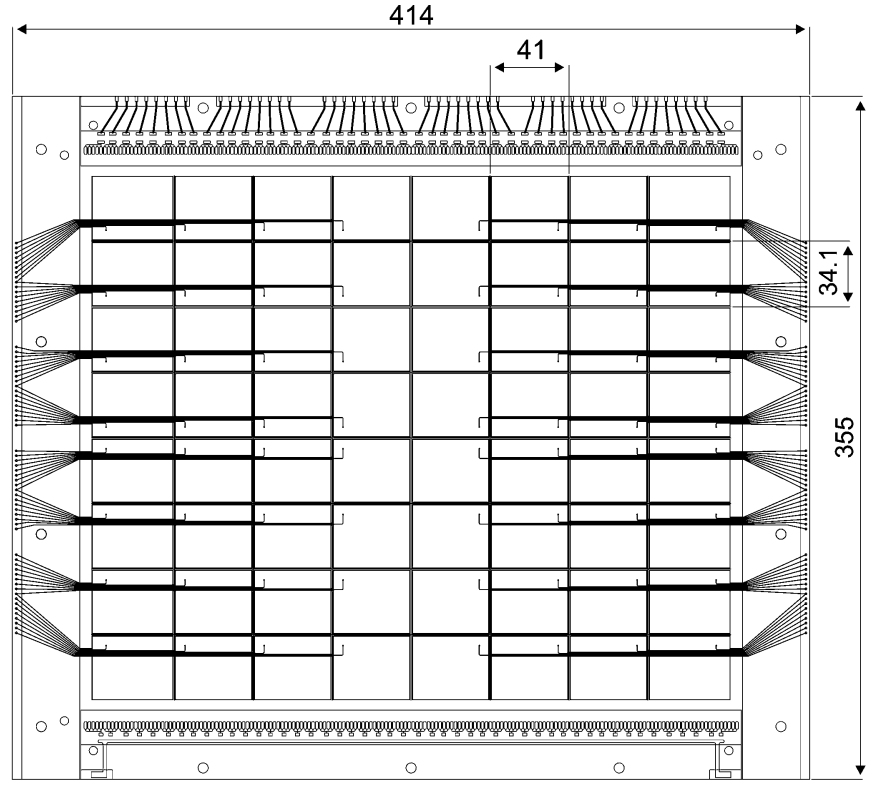

Fig. 2. Readout scheme of the anode wires signals from the top and of the cathode pads signals from the left and right sides of the chamber.

The panels are made of polyurethane foam injected in between two FR4 printed circuit boards. On each circuit, the pad structure is printed on one side and the signal tracks on the other side, with Ni/Au plating on the $35 \mu \mathrm{m}$ thick $\mathrm{Cu}$ clad. For regions R1 and R2, both cathodes and anodes are read-out. Cathodes are divided in 64 pads separated by a ground line. The wires are connected together in groups of different sizes defining 48 wire pads per gap. Fig. 2 shows the two sides of a typical circuit board (M3-R1) and the read-out scheme of the cathode and anode pads. For M2-R1 and M3-R1, it has been shown that the signal-to-noise ratio is improved when the signal is read-out from the two cathodes defining a gap in a hardwired OR (double cathode readout) [4].

Two wire soldering bars are glued to the panels before wiring so that the wires are soldered at the correct half-gap distance. The anode signal is collected on one of the soldering bars. Highvoltage resistors and decoupling capacitors are soldered directly to these bars, inside the gas volume. Gas tightness is obtained with a natural rubber O-ring. The panels are kept together by an aluminum mechanical frame on which the Faraday cage is fixed. The aluminum is alodined for better electrical contact in the long term.

The five main production steps on wired panels are: ) bar glueing; 2) wire winding and wire glueing; 3 ) automatic wires
TABLE II

MAIN SPECIFICATIONS FOR THE MWPC OF THE LHCb MUON SYSTEM

\begin{tabular}{|l|ll|}
\hline Panel Thickness & $9070 \mu \mathrm{m}$ & $\pm 70 \mu \mathrm{m}$ \\
\hline Wire pitch & $2000 \mu \mathrm{m}$ & $\pm 50 \mu \mathrm{m}$ \\
\hline Side bars thickness & $2500 \mu \mathrm{m}$ & $\pm 50 \mu \mathrm{m}$ \\
\hline \hline Wire tension & $50 \mathrm{gr}<\mathrm{T}_{0}<150 \mathrm{gr}$ & $\pm 10 \% \mathrm{~T}_{0}$ \\
\hline Chamber Thickness & $<7500 \mathrm{~mm}$ \\
\hline HV dark current & $<50 \mathrm{nA}$ at $2.9 \mathrm{kV}(4$ gaps $)$ \\
\hline Gas leak rate & $<0.2 \% \mathrm{~h}^{-1}$ at 5 mbar overpressure \\
\hline
\end{tabular}

soldering; 4) components and connectors soldering; and 5) chamber assembly. The production of the other panels is very simple. A kapton foil is glued to the areas close to the high-voltage components and the connectors are soldered. Gas inlets are also glued to the corners of the outer panels. The overall procedure is described in [1].

The MWPC response uniformity is mainly determined by the mechanical precision of the assembly. Mechanical tolerances have been derived from simulations [5] with the requirement of $30 \%$ maximum variation of the gas gain over the surface. This allows working only $50 \mathrm{~V}$ over the beginning of the plateau while keeping the efficiency above $99 \%$ over the whole active surface. Mechanical tolerances are shown on Table II, together with other specifications. The final specification on the working chambers concerning the response uniformity requires that the gas gain should not vary by more than $30 \%$ for at least $95 \%$ of the surface.

\section{QUALITY CONTROL AND QUALITY ASSURANCE}

In order to guarantee a constant quality of the produced chambers and to allow fast tracing of potential production problems, precise procedures are defined for each production step and $\log$ sheets are filled in after each step. In addition, several quality tests are performed at different stages of the production. A chamber is only accepted for installation in the experiment if the results of the tests are within the specifications shown in Table II.

The six main quality tests are as follows.

1) The height of the wire soldering bars, corresponding to half the gap thickness, is measured with a comparator at all the positions of the assembly holes along the bars. The glueing precision, defined as the RMS of the half-gap measurement distribution, is better than $20 \mu \mathrm{m}$.

2) The wire pitch is measured using two charge-coupled device cameras connected to telecentric lenses. With this optics, the magnification factor does not depend on the object distance. The two cameras, one at each side of the panel, are precisely moved by $2 \mathrm{~mm}$ steps over the wires and the recorded images are processed to recognize the wires and fit the distance between them. The precision on the pitch is about $17 \mu \mathrm{m}$, well within the specification.

3) The tension on each wire is evaluated by measuring its resonance frequency for mechanical oscillations [6]. The wire is forced to oscillate by a periodic high voltage (700 V) applied to a rigid sensor wire positioned $1 \mathrm{~mm}$ above the wire plane. This oscillation produces a variation of the capacitance between the two wires which is measured in a short time window in phase with the excitation. A plot 


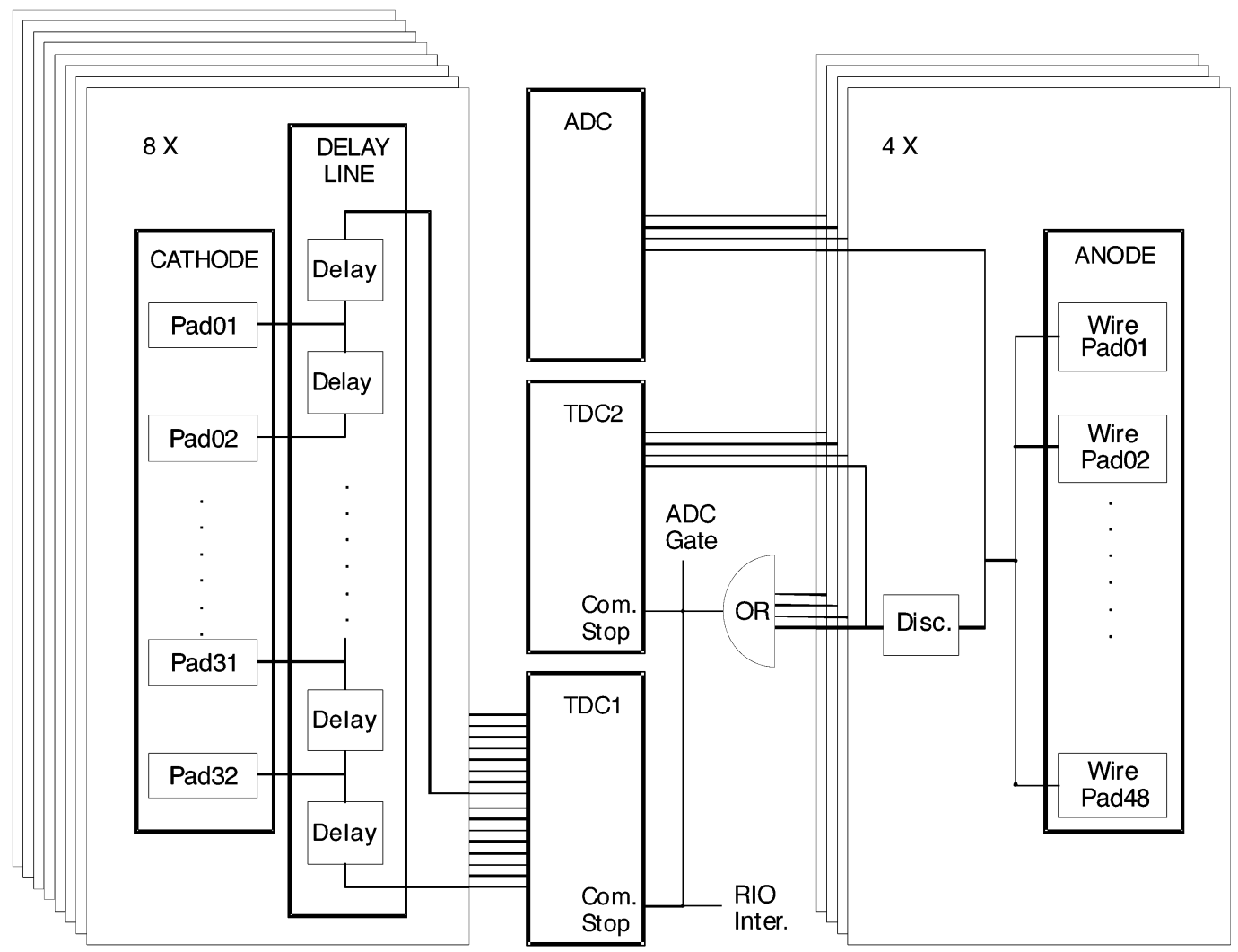

Fig. 3. Schematic view of the electronics setup used for the gas gain uniformity measurement.

of this quantity as a function of the excitation frequency shows a typical excitation curve. The tension is related to the fundamental frequency $f$ according to the simple relation $T=\rho \cdot(2 L \cdot f)^{2}$, where $\rho$ and $L$ are respectively the wire density and length. We obtain a wire tension of $67 \pm 2 \mathrm{~g}$, well below the elasticity limit of the wire $(\sim 150$ g).

4) The chamber gas leak rate is measured by comparing of the overpressure in a closed chamber $\Delta P_{\mathrm{ch}}$, with the overpressure in a sealed reference volume $\Delta P_{\text {ref }}$. The difference in temperature between the chamber and the reference volume is maintained constant by placing them in a thermally isolated box. In these conditions, the relative leak rate, expressed in percent of the volume per time unit, is simply given by the slope of the curve $\left(\Delta \mathrm{P}_{\text {ch }}-\Delta \mathrm{P}_{\text {ref }}\right) / \mathrm{P}_{\mathrm{atm}}$. The average leak rate obtained is about $0.05 \%$ per hour at 5 mbar overpressure (which corresponds approximately to $1 \mathrm{mbar} \mathrm{l} / \mathrm{h}$ for an M3-R1 chamber).

5) After the high-voltage training, including several hours of negative high voltage, the dark current is measured at $2.9 \mathrm{kV}$. Then, the chamber is exposed to a $640 \mathrm{GBq}$ ${ }^{137} \mathrm{Cs}$ source in order to check for the absence of persistent (Malter like) currents when the source is switched off.

6) For the gas gain uniformity measurement we developed an original method which is described in details in Section IV. It combines time spectrum analysis for pad identification with pulse height analysis to determine a gas gain map of the chamber. It has been introduced as an alternative to the method of the ionization current measurement when the chamber is exposed to a $370 \mathrm{MBq}$, collimated, ${ }^{137} \mathrm{Cs}$ source scanned over the chamber surface. The latter method suffers from significant border effects and is not adapted for the small chambers produced at CERN.

Furthermore, several aging tests have been performed at the ENEA Casaccia Caloppe facility [4] and at the CERN gamma irradiation facility [7]. Linear charges of up to $0.5 \mathrm{C} / \mathrm{cm}$ have been accumulated, corresponding to five years of $\mathrm{LHCb}$ operation. The observed aging effects led to the conclusion that these chambers are acceptable for operation in the LHCb experiment.

\section{Gas Gain Uniformity MEASUREMENT}

Our gas gain uniformity measurement is based on the analysis of the spectrum obtained when the chamber is exposed to a ${ }^{241} \mathrm{Am}$ radioactive source. This spectrum exhibits a peak in a position proportional to the gas gain. This peak, which is used as a reference peak, results mainly from the absorption of fluorescence X-rays emitted by the copper atoms of the cathode plane when excited by the $60 \mathrm{keV}$ gamma rays coming from the source. It is degraded by the relatively poor energy resolution of the chamber and the superposition with the Compton spectrum of the $60 \mathrm{keV} \gamma$-rays. The gamma activity of the source (24 MBq) is low enough to minimize radiation safety issues. Moreover, the source can stay in a fixed position which allows illumination of the whole chamber.

The gas gain variation over a gap surface is given by the variation of the reference peak position in the spectra taken at each of the 64 cathode pads. In order to obtain a spectrum for each 


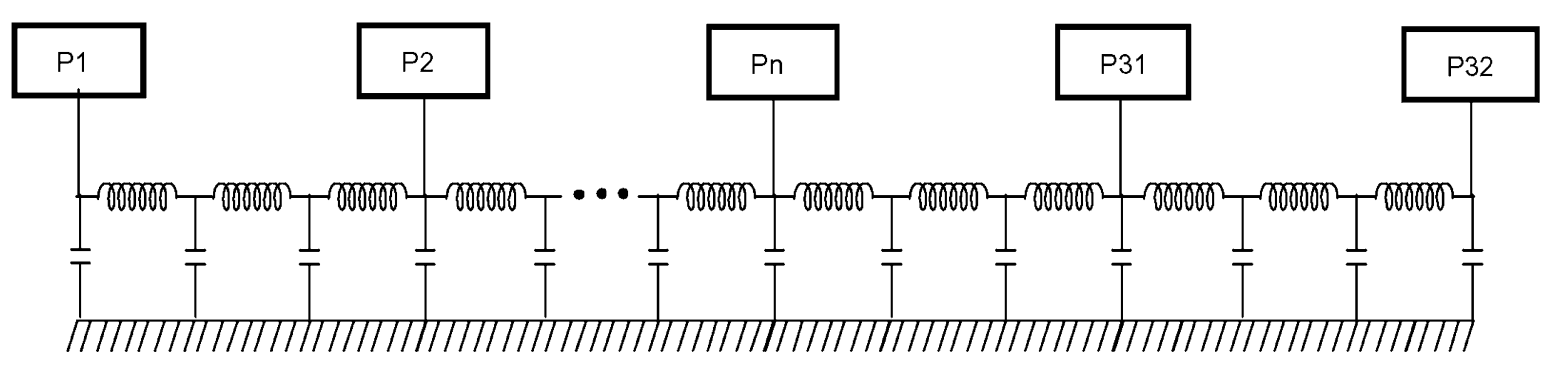

Fig. 4. Delay-line circuit showing the pad connections at each three delay cells. The capacitances connected to ground in the positions where pads are connected represent the pad capacitances themselves.

pad without having to equip $4 \times 64$ amplifiers and ADC channels, the delay-line technique is used. Signals from the different pads of a single gap are sent as input to different cells of a discrete delay-line. The pad position is unambiguously associated to the time delay between the two outputs of the delay-line. Two delay-lines are needed to read out all the pads of a single gap (Fig. 2). The anode wire pads of the gap are connected together and the resulting signal is amplified and splitted in two: one is sent to an ADC and the other one is discriminated. The signals from the four anode wire planes are logically OR-ed to form a common stop signal for the TDCs and the gate for the ADC. The electronics setup is illustrated in Fig. 3. The analog part is installed close to the chamber, while the TDCs and ADCs are implemented with VME modules. In the off-line analysis, simple time window selection in the TDC allows the identification of the active pad and the production of the corresponding ADC spectrum. A two-dimensional plot of the peak position versus the cathode pad position provides an image of the spatial distribution of the gas gain.

\section{A. Delay-Line Circuit Description}

The value of the inductances and capacitances of the delayline circuits must be chosen according to the characteristics of the detector, the data acquisition environment and the commercial availability of the electronics components. The cathode pads have a nonnegligible capacitance to ground which imposes a minimum value for the delay-line capacitances. To ensure good linearity and to reduce signal deformation along the line, the pad capacitances must be integrated to the delay-line, i.e., whenever a pad is connected to a delay cell, no discrete capacitance is used. A large cutoff frequency $\omega_{0}=1 / \sqrt{L / C}$ is required to cope with the fast signal from the chamber. The characteristic impedance $(Z \cong \sqrt{L / C})$ should be as high as possible, so that the signal amplitude is not reduced by differentiation. All these requirements are matched by a circuit with 120 cells of aproximately $8 \mathrm{~ns}$ delay, implemented with $C=39 \mathrm{pF}$ and $L=1.5 \mu \mathrm{H}$ (Fig. 4). Two consecutive pads are separated by three delay cells, i.e., $24 \mathrm{~ns}$ in time, while the intrinsic time resolution of the chamber is approximately $4 \mathrm{~ns}$.

\section{B. Amplifier Circuit Description}

The amplifier circuit must feature high bandwidth, high gain, and low noise. In order to achieve these characteristics it is designed with five stages. The first stage uses a field effect transistor (F245A) [8] introduced to ensure a high input impedance. Three wide-band low-gain intermediate stages are implemented

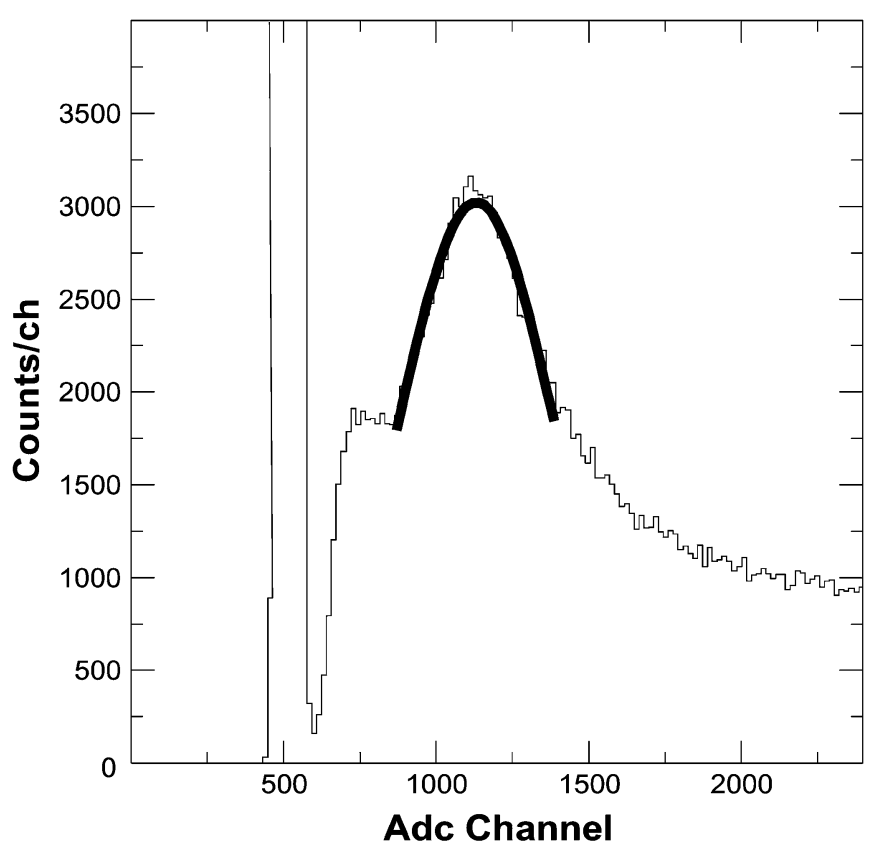

Fig. 5. Typical ADC spectrum showing the fit of a Gaussian curve to the reference peak.

with bipolar transistors (PN2369A) [9]. The last stage is simply a common-collector current driver.

\section{Results}

A typical ADC spectrum is shown in Fig. 5, with a fit to the reference peak position. In order to compare the gain from one gap to the others, the reference peak position is normalized to the signal of a precision pulse generator injected at the level of the amplifier input. This quantity is called normalized gain. A typical TDC spectrum is shown in Fig. 6. It shows the distribution of time differences between the signals coming from the two outputs of a delay-line. An amplified view of the peaks, corresponding to two adjacent pads, is also shown. The time range defined by the lines around each peak selects the events that will compose the ADC spectrum corresponding to that pad. It is worth noting that the uniformity of the distribution of the number of events in the TDC spectrum peaks provides information about possible localized defects inside the chamber.

A typical final result for an outer gap is shown in Fig. 7. It is a map of the normalized gain as a function of the pad number. The gas gain is slightly lower at the center of the gap. The distribution is usually flat for central gaps. This is explained by the bending of the panel caused by the mechanical force applied to 


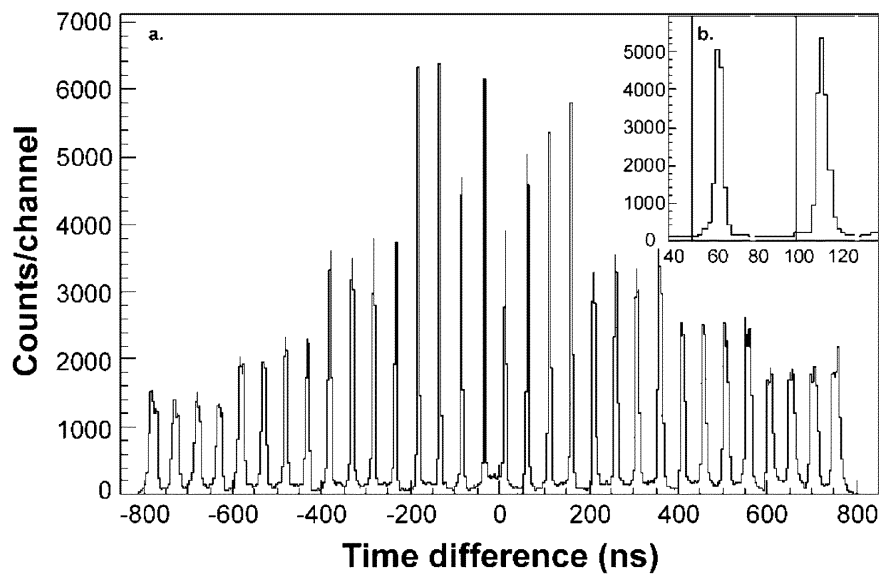

Fig. 6. (a) TDC spectrum with all the 32 peaks corresponding to one side of one chamber gap. (b) Amplified view showing the peaks coresponding to two adjacent pads. The lines mark the time ranges used to select the pads.

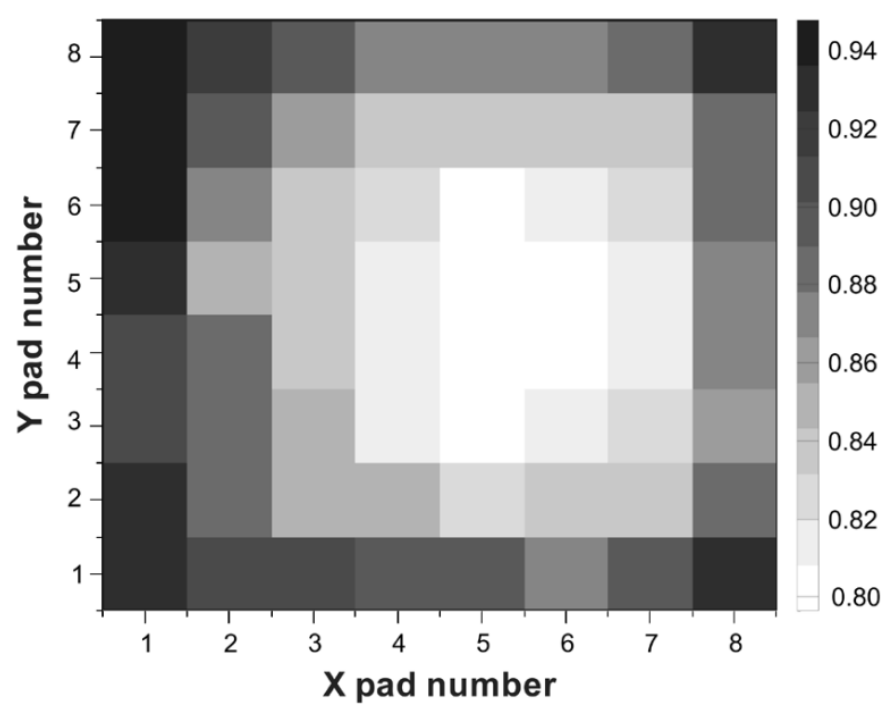

Fig. 7. Typical two-dimensional distribution of the normalized gain in an outer gap of a LHCb MWPC chamber (M3-R1).

the o-ring. Overpressure in the chamber is very low and has a negligible effect.

The distribution of the normalized gas gain for all M3-R1 chambers is shown in Fig. 8. The RMS of the distribution is $10 \%$ and all measurements are well within the specified $30 \%$ of the mean value.

\section{Conclusion AND Perspectives}

CERN is producing MWPCs for the inner regions of the $\mathrm{LHCb}$ Muon system. The production line meets all the specifications with a production rate of two chambers per week. The quality assurance program, along with the extensive tests performed on the chambers is a guarantee for their long-term reliability. An original gas gain uniformity measurement method, free of border effects, has been developed using a fixed, low-intensity radioactive source. It has been used to demonstrate that the gas gain uniformity over the surface is better than $30 \%$.

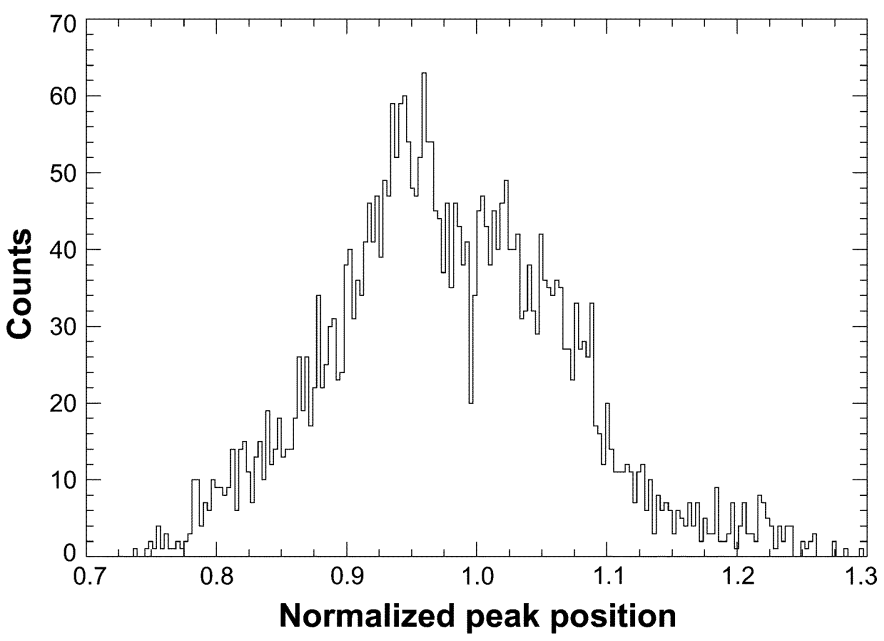

Fig. 8. Distribution of the normalized gas gain for the MWPC chambers produced at CERN for the region M3-R1 of the LHCb Muon System. The average of the distribution is 0.98 with a RMS value of 0.09 .

A standalone system with a real-time data acquisition is currently under tests. It is based on the techniques reported in the present work and is implemented in a single board containing one eight-channel TDC chip and two two-channel ADCs. All the logics circuitry is concentrated in one FPGA circuit, which communicates to a personal computer via the parallel or USB port. As a perspective for the present work, the standalone system should allow the automatization of the whole chamber characterization process, from the data acquisition to the production of the final report.

\section{REFERENCES}

[1] CERN, "LHCb muon system technical design report: LHCb Collaboration," CERN, Geneva, Switzerland, CERN-LHCC 2001-010, May 2000.

[2] CERN, "Second addendum to the LHCb muon system technical design report: LHCb Collaboration," CERN, Geneva, Switzerland, CERNLHCb-2004-116, Mar. 2005.

[3] W. Bonivento et al., "Development of the CARIOCA front-end chip for the LHCb muon detector," Nucl. Instrum. Methods, vol. 491, pp. A233-A243, Sept. 2002.

[4] G. Lanfranchi et al., "Time resolution and aging properties of the MWPC's for the LHCb muon system," Nucl. Instrum. Methods, vol. 535, pp. A221-A225, 2004.

[5] W. Riegler, "Detector physics and performance simulations of the MWPC for the LHCb muon system," CERN, Geneva, Switzerland, LHCb-2000-060, Aug. 2000.

[6] P. Ciambrone et al., "Automated wire tension measurement system for LHCB muon chambers," Nucl. Instr. Meth. Phys. Res. A. in press.

[7] V. Suvorov, T. Schneider, B. Schmidt, W. Riegler, A. Kashchuk, and D. Hutchcroft, "First results of an aging test of a full scale MWPC prototype for the LHCb muon system," Nucl. Instrum. Methods, vol. 515, pp. A220-A225, 2003.

[8] Philips. (1996, Jul.) N-channel silicon field-effect, transistors. Philips Semiconductors, Eindhoven, The Netherlands. [Online]. Available: http://www.semiconductors.philips.com/acrobat_download/datasheets/ BF245A-B-C_2.pdf.

[9] Philips. (1999, Apr.) NPN switching transistors. Philips Semiconductors, Eindhoven, The Netherlands. [Online]. Available: http://www.semiconductors.philips.com/acrobat_download/datasheets/ PN2369_2369A_3.pdf. 